NOTE: This is a preprint of a paper being submitted for publication. Contents of this paper should not be quoted or referred to without permission of the author(s).

To be submitted to The Sixteenth Intemational Conference on Atomic Collisions in Solids, Johannes Kepler Universitaet Linz, Linz, Austria, July 17-21, 1995

The submitted manuscript has been authored by a contractor of the U.S. Government under contract No. DE-ACO5-840R21400. Accordingly, the U.S. Government retains nonexclusive, royalty-free license to publish or reproduce the published form of this reproduce the published form of this Government purposes."

\title{
BINDING ENERGY EFFECTS IN CASCADE EVOLUTION AND SPUTTERING
}

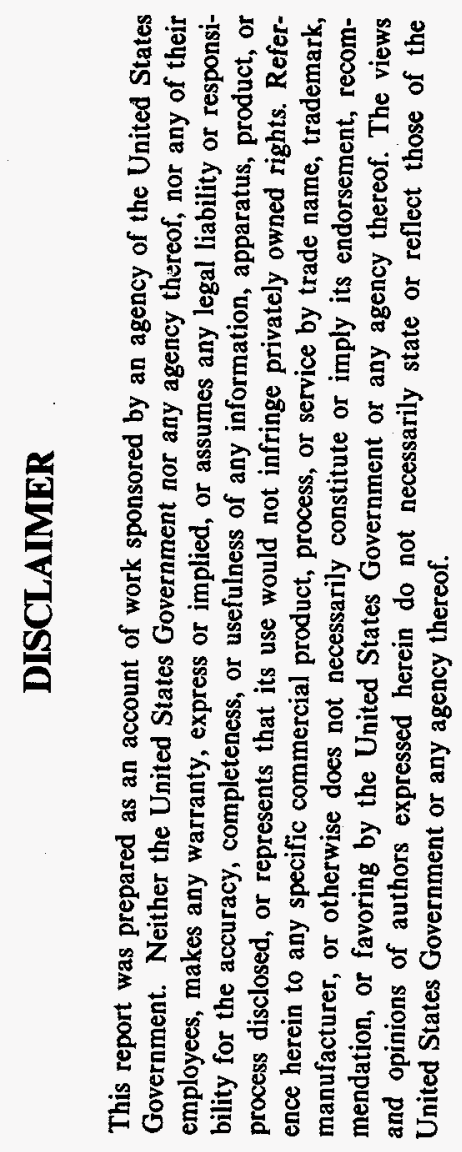

Mark T. Robinson

\author{
SOLID STATE DIVISION \\ OAK RIDGE NATIONAL LABORATORY \\ Managed by
}

MARTIN MARIETTA ENERGY SYSTEMS, INC.

Under

Contract No. DE-AC05-84OR21400

With the

U. S. DEPARTMENT OF ENERGY

OAK RIDGE, TENNESSEE

June 1995 


\title{
Binding Energy Effects in Cascade Evolution and Sputtering
}

\author{
Mark T. Robinson \\ Solid State Division, Oak Ridge National Laboratory, \\ P. O. Box 2008, Oak Ridge, Tennesseє, 37831-6032, U. S. A.
}

\begin{abstract}
The MARLOWE model was extended to include a binding energy dependent on the local crystalline order, so that atoms are bound less strongly to their lattice sites near surfaces or associated damage. Sputtering and cascade evolution were studied on the examples of self-ion irradiations of $\mathrm{Cu}$ and $\mathrm{Au}$ monocrystals. In cascades, the mean binding energy is reduced $\sim 8 \%$ in $\mathrm{Cu}$ with little dependence on the initial recoil energy; in $\mathrm{Au}$, it is reduced $\sim 9 \%$ at $1 \mathrm{keV}$ and $\sim 15 \%$ at $100 \mathrm{keV}$. In sputtering, the mean binding energy is reduced $\sim 8 \%$ in $\mathrm{Cu}$ and $\sim 15 \%$ in Au with little energy dependence; the yields are increased about half as much. Most sites from which sputtered atoms originate are isolated in both metals. Small clusters of such sites occur in $\mathrm{Cu}$, but there are some large clusters in $\mathrm{Au}$, especially in $\{111\}$ targets. There are always more large clusters with damage-dependent binding than with a constant binding energy, but only a few clusters are compact enough to be regarded as pits.
\end{abstract}

\section{Introduction}

Computer simulation models of displacement cascade evolution based on the binary collision approximation $(\mathrm{BCA})$ often ignore the attractive interactions that bind atoms into solids or approximate them by a binding energy independent of the local crystalline order or of the 
reduced number of neighbors of surface atoms. Both nearby surfaces and adjacent defects should influence the ease of displacing atoms, but the quantitative effect is uncertain, since it depends on the overall cascade statistics. Yamamura [1] has used a damage-dependent surface binding energy in his mixed code DYACAT for studies of sputtering by cluster ions. The objective of the present study was to consider the extent to which such features need be included in $\mathrm{BCA}$ modelling. This is done by cascade evolution and sputtering calculations using the well-known MARLOWE program [2-6].

\section{The Computational Model}

MARLOWE was enhanced to use binding energies varying with the number of occupied adjacent lattice sites: atoms near target surfaces or in regions disordered by accompanying damage are bound less strongly. In other respects, the computational model was that described earlier [2-6]. Studies were made of the sputtering of $\{001\}$ and $\{111\} \mathrm{Cu}$ and $\mathrm{Au}$ crystals by normally incident 1 - to $100-\mathrm{keV}$ self-ions and of the evolution of displacement cascades in the bulk metals. Except for the binding model, the parameters were the same as in a previous study [6] of the statistics of sputtering: the targets are fcc crystals with lattice constants $0.3615 \mathrm{~nm}$ in $\mathrm{Cu}$ and $0.4078 \mathrm{~nm}$ in Au. The Moliére potential was used with the screening lengths $7.38 \mathrm{pm}$ in $\mathrm{Cu}$ and $7.50 \mathrm{pm}$ in $\mathrm{Au}$. Collisions in the developing cascades were ordered in time, stopped cascade atoms were not included as targets, and all targets were static. A local inelastic energy loss was employed [7], normalized to the well-known LSS theory [8]. Reported results are based on sets of 1000 primary particles. In sputtering calculations, the initial impact points were distributed uniformly over the unit mesh on the crystal surface; in cascade calculations, the initial directions were distributed uniformly in a cubic zone.

In the MARLOWE model, the total binding energy of an atom is divided into two parts: one, $E_{b}$, is local to each lattice site; the other, $E_{s}$, is applied to particles passing through the target surface. The extension is to write 


$$
E_{b}(n)=E_{b}^{\prime \prime}+(n / N)^{\ell}\left(E_{b}^{\prime}-E_{b}^{\prime \prime}\right)
$$

where $N$ is the total number of neighboring sites, $n$ is the number of such sites occupied by atoms, $E_{b}^{\prime}$ and $E_{b}^{\prime \prime}$ are the maximum and minimum local binding energies, and $\ell$ is either 1 or

$\frac{1}{2}$. In materials with ionic or covalent bonding, $\ell=1$ is appropriate, but for metals, $\ell=\frac{1}{2}$ is a better choice $[9,10]$. A lattice site is considered occupied if it has not been disturbed: if it is the site of a replacement event; or if it is the site in a close pair, defined in MARLOWE as a stopped cascade atom whose closest lattice site is vacant [3]. The binding energy $E_{b}^{\prime \prime}$ is used when a nonlattice target atom is displaced, a feature not needed in the present calculations. Finally, in replacement events in which a projectile replaces a displaced target atom on its lattice site, the binding energy $E_{b}^{\prime \prime \prime}$ is used instead. Normally, $E_{b}^{\prime \prime \prime} \ll E_{b}^{\prime}$ is chosen so that the lengths of long sequences of replacements accord with molecular dynamics calculations. There are now five parameters in the MARLOWE binding model: $E_{b}^{\prime}, E_{b}^{\prime \prime}, E_{b}^{\prime \prime \prime}, E_{s}$, and $\ell$. These offer considerable choice, usually subject to the constraint $E_{b}^{\prime}+E_{s}=2 U$, where $C^{\prime}$ is the cohesive energy of the target, $3.5 \mathrm{eV}$ for $\mathrm{Cu}$ and $3.8 \mathrm{eV}$ for Au. Neglecting the effects of relaxation, the vacancy formation energy is $E_{v}=\left(1-2^{-\ell}\right)\left(E_{b}^{\prime}-E_{b}^{\prime \prime}\right)$. Experimentally, $E_{v}$ is $1.28 \pm 0.05 \mathrm{eV}$ in $\mathrm{Cu}$ and $0.93 \pm 0.04 \mathrm{eV}$ in $\mathrm{Au}[11]$

The calculations reported compare two models: in the constant binding model, $E_{b}$ is independent of the number of vacant sites and had the value $U$ for each target. The damagedependent binding model was used with $E_{b}^{\prime}=U, E_{b}^{\prime \prime}=0.2 \mathrm{eV}, N=12$, and $\ell=1$, a choice that maximizes the effects of the model, although $\ell=\frac{1}{2}$ is preferred for metallic targets; the unrelaxed $E_{v}=1.65 \mathrm{eV}$ for $\mathrm{Cu}$ and $1.8 \mathrm{eV}$ for $\mathrm{Au}$ (if $\ell=\frac{1}{2}, E_{v}=0.97$ and 1.05 $\mathrm{eV}$, respectively). In all cases, $E_{b}^{\prime \prime \prime}=0.2 \mathrm{eV}$ and, where required, the value of $E_{s}=U$. Recoiling atoms were followed until they left a target surface or until their kinetic energies fell below $E_{c}=U$. Escaping atoms were required to surmount a planar surface-binding barrier to be regarded as sputtered. It should be observed that the lowered binding energies of surface atoms are offset in sputtering to the extent that they are ejected from the surface in replacement events. 


\section{Sputtering Yields and Binding Energies}

Fig. 1 shows self-sputtering yields calculated for 1 - to $100-\mathrm{keV}$ atoms normally incident on $\{001\}$ and $\{111\}$ surfaces of $\mathrm{Cu}$ and $\mathrm{Au}$ monocrystals using both binding models. The statistical uncertainties of the individual results are shown in the figure. These reflect the width of the distribution of individual ion yields, which becomes very broad at the higher energies. The yield is higher with damage-dependent binding, but differences between the models are small, sometimes not exceeding the statistical uncertainties. This result can be understood by examining the spectrum of binding losses, or equivalently, the frequency with which atoms are displaced from sites with various numbers of unoccupied neighbor sites: the loss of an atom from one nearest neighbor site corresponds to a change in $E_{b}$ of $\sim 8 \%$.

Fig. 2 shows the fraction of atoms displaced from sites with $m$ vacant neighbors for 1 - and $100-\mathrm{keV}$ cascades in $\mathrm{Cu}$ and $\mathrm{Au}$ crystals. Fewer than $\sim 5 \%$ of the $\mathrm{Cu}$ atoms are displaced from sites with more than two vacant neighboring sites and this fraction hardly changes with increasing primary kinetic energy. The mean number of vacant neighbor sites, $\langle m\rangle$, is $\sim 1$. independent of energy. The fraction of displacements from Au sites with more than two vacant neighbors. in contrast, rises from $8 \%$ at $1 \mathrm{keV}$ to nearly $25 \%$ at $100 \mathrm{keV}$ and $<m>$ rises from just over 1 to nearly 2 in the same energy range. Nevertheless, the energy dependence of the distributions is not dramatic: they are dominated, whatever the initial kinetic energy, by the large number of recoils produced not far above the cut-off energy $E_{c}$. Because the mean number of vacancies adjacent to a displacement site is small, the mean binding energy is only a little smaller than the value in the constant binding model.

Fig. 3 shows similar data for sputtering calculations. An added feature here is the second peak in the $1-\mathrm{keV}$ distributions. This contains atoms displaced from surface sites: atoms in perfect $\{001\}$ and $\{111\}$ surfaces of fcc metals lack 4 and 3 nearest neighbors, respectively. As the primary energy increases, the surface peak gradually disappears, engulfed in the many displacements from interior sites. Unlike the cascade results, $\langle m\rangle$ hardly changes in either metal over the $1-$ to $100-\mathrm{keV}$ energy range: it is $\sim 1$ in $\mathrm{Cu}$ and nearly $2 \mathrm{in} \mathrm{Au}$. 
Again the mean binding energy is reduced only a little from the maximum value. The disappearance of the surface peak reflects the low efficiency of the sputtering process: the fraction of displaced atoms which are sputtered exceeds $10 \%$ only for the Au $\{111\}$ target at $1 \mathrm{keV}$ and decreases rapidly with increasing initial primary energy. Furthermore, roughly half the sputtered atoms are produced in replacement events in which the binding energy is not damage-dependent.

\section{Clusters of Sputter Sites}

The clustering of lattice sites from which sputtered atoms originate was also examined. Clusters are defined by nearest-neighbor links: to be considered part of a cluster, a site must have at least one nearest neighbor site from which a sputtered atom originated. Fig. 4 shows the fraction of all sputter sites in clusters of more than $n$ sites in $100 \mathrm{keV}$ self-sputtering. In $\mathrm{Cu}$, very few sites are in clusters of more than two or three, there is little difference between the two crystal orientations, and not much between the binding models, although the $\{111\}$ crystals show more clusters of five to ten members with damage-dependent binding. In $\mathrm{Au}$, on the other hand, large clusters are found, especially with $\{111\}$ targets and damagedependent binding. This behavior reflects the higher density of cascades in Au as compared with $\mathrm{Cu}$. Nonetheless, isolated sputter sites and small clusters of two to five sites are always much the most probable.

Large clusters may be related to the pits observed experimentally in the sputtering of heavy metals by heavy ions [12], but they need more than mere size to fill this rôle: they must also be reasonably compact. As a measure of compactness, define

$$
\hat{z}=\frac{1}{2(n-1)} \sum_{j=1}^{n} \ell_{j}-1
$$

where $n$ is the number of sites in a cluster and $\ell_{j}$ counts the links between the $j$ th site and its neighbors. $\hat{z}$ is a measure of the number of links in a cluster in excess of the minimum number $2(n-1)$ : it is 0 for tree-like clusters without loops and increases as the cluster 
becomes more compact to a limit depending on the geometry. In the present calculations, clusters with $\hat{z}$ slightly over 1 were occasionally observed. Note from the definition that the value of $\hat{z}$ is always a common fraction with denominator $n-1$.

Fig. 5 shows the frequency distribution of $\hat{z}$ for $20 \mathrm{keV}$ self-sputtering of $\mathrm{Au}\{111\}$ surfaces. Separate distributions are shown for clusters in three different size ranges, normalized to the total number of clusters observed. Common fractions can be assigned to each of the observed frequency groups in the figure. This is done explicitly in the lowest panel. Such assignments are limited by the resolution in scoring the histograms and the coincidences between certain values. The fractions are related to the geometry of the clusters, but it is difficult to assign structures uniquely to large clusters. A cluster of six sites with $\hat{z}=\frac{6}{5}$ can be constructed from two tetrahedra of lattice atoms sharing an edge, for example. This compact cluster appears in Fig. 5. Two clusters with $\hat{z}=1$ can be envisioned: a tetrahedron of four sites or a planar arrangement of one site and its six nearest neighbors on a $\{111\}$ plane fit this description. Both are compact, but cannot be separated in statistical data like Fig. 5.

\section{Discussion}

The effects of damage-dependent binding on the sputtering yield are diluted for two reasons. First, an atom escaping from the crystal in the MARLOWE model must surmount not only $E_{b}$. but also $E_{s}$, and with the parameters used in the present calculations, the latter halves the relative effect of changes in the former. Second, as noted earlier, those atoms which are ejected following replacement events surmount only the much smaller $E_{b}^{\prime \prime \prime}$, which is not affected by damage or surfaces. However, the binding model also affects the number of cascade atoms produced and thus influences the number, energy, and angular distribution of atoms reaching the surface. No data are currently available that reflect directly on this point, but it is likely that such flux changes partly offset the diluting effects of the surface binding energy. 
The damage-dependent binding model illustrated here is a type of nonlinearity that is easily added to a BCA simulation. Quantitatively, the effect of this model on sputtering yields is not large, but it produces more large clusters of sputter sites, especially in dense targets like Au. The densities of large compact sputter site clusters are small, but appear consistent with the experimental density of $\sim 1 \%$ [12]. Better statistics on such clusters can be achieved, but only at the cost of increased computer time.

The damage-dependent binding model can be extended to include the effects of stopped interstitial cascade atoms as well as vacancies. It should also be feasible to elaborate the surface binding model along similar lines.

\section{Acknowledgements}

Research sponsored by Division of Materials Science, U. S. Department of Energy, under contract No. DE-AC05-84OR21400 with Lockheed Martin Energy Systems, Inc. 


\section{REFERENCES}

[1] Y. Yamamura, Nucl. Instr. and Meth. B 33 (1988) 493.

[2] M. T. Robinson, Phys. Rev. B 40 (1989) 10717.

[3] M. T. Robinson, Nucl. Insr. and Meth. B $48(1990) \leftarrow 08$.

[4] M. T. Robinson, Radiat. Eff. 130/131 (1994) 3.

[5] M. T. Robinson, User's Guide to MARLOWE (Version 13c), unpublished: this version of the code is available from the Radiation Shieldirg Information Center, Oak Ridge National Laboratory, P. O. Box 2008, Oak Ridge, Ternessee 37831-6362, U.S.A. (e-mail: pdc@ornl.gov; http://epicws.epm.ornl.gov).

[6] M. T. Robinson, Nucl. Insr. and Meth. B 90 (1994) 509.

[7] O. S. Oen and M. T. Robinson. Nucl. Instr. and Me:b. 132 (1976) 647.

[8] J. Lindhard and M. Scharff, Phys. Rev. 124 (1961) 128.

[9] A. E. Carlsson, Solid State Physics 43 (1990) 1.

[10] H. Gades and H. M. Urbassek. Nucl. Instr. and Methods B 69 (1992) 232.

[11] P. Erhart, in: Atomic Defects in Metals ed. by H. Illmaier, Landholt-Börnstein, New Series, Group III, Vol. 25 (Springer, Berlin, 1991) pp. 202-263.

[12] K. L. Merkle and W. Jäger, Phil. Mag. A 44 (1981; 741. 


\section{FIGURES}

FIG. 1. Self-sputtering yields in $\{001\}$ and $\{111\} \mathrm{Cu}$ and $\mathrm{Au}$ monocrystals for 1 - to $100 \mathrm{keV}$ primary particles. Yields are shown for both constant and damage-dependent binding. The lines are drawn merely to guide the eye.

FIG. 2. The fraction of displacements from sites with $m$ vacant nearest neighbors, for cascades in $\mathrm{Cu}$ and $\mathrm{Au}$ monocrystals generated by 1 - and $100 \mathrm{keV}$ primary recoils, using damage-dependent binding. The mean value $\langle m\rangle$ is shown for each case. To guide the eye, the points in the figure are connected by straight line segments.

FIG. 3. The fraction of displacements from sites with $m$ vacant nearest neighbors, during the self-sputtering of $\{001\}$ and $\{111\} \mathrm{Cu}$ and $\mathrm{Au}$ monocrystals generated by 1 - and $100-\mathrm{keV}$ primary particles, using damage-dependent binding. The mean value $\langle m\rangle$ is shown for each case. To guide the eye, the points in the figure are connected by straight line segments.

FIG. 4. The fraction of sputter sites in clusters of size $>n$. for the self-sputtering of $\mathrm{Cu}$ and Au monocrystals at $100 \mathrm{keV}$. comparing constant and damage-dependent binding. The lines are drawn just to guide the eye.

FIG. 5. The distribution of the mean bond number $\hat{z}$ in clusters of sputter sites, for $20 \mathrm{keV}$ self-sputtering of $\mathrm{Au}\{111\}$ surfaces, using damage-dependent binding. The frequencies, normalized to one cluster, are shown for three ranges of cluster size $n$. The average cascade contained 17.25 \pm 0.39 sputter sites. Common fractions assignable to the cluster groups are shown in the lower panel. 
ORNL-DWG 95M-55T7

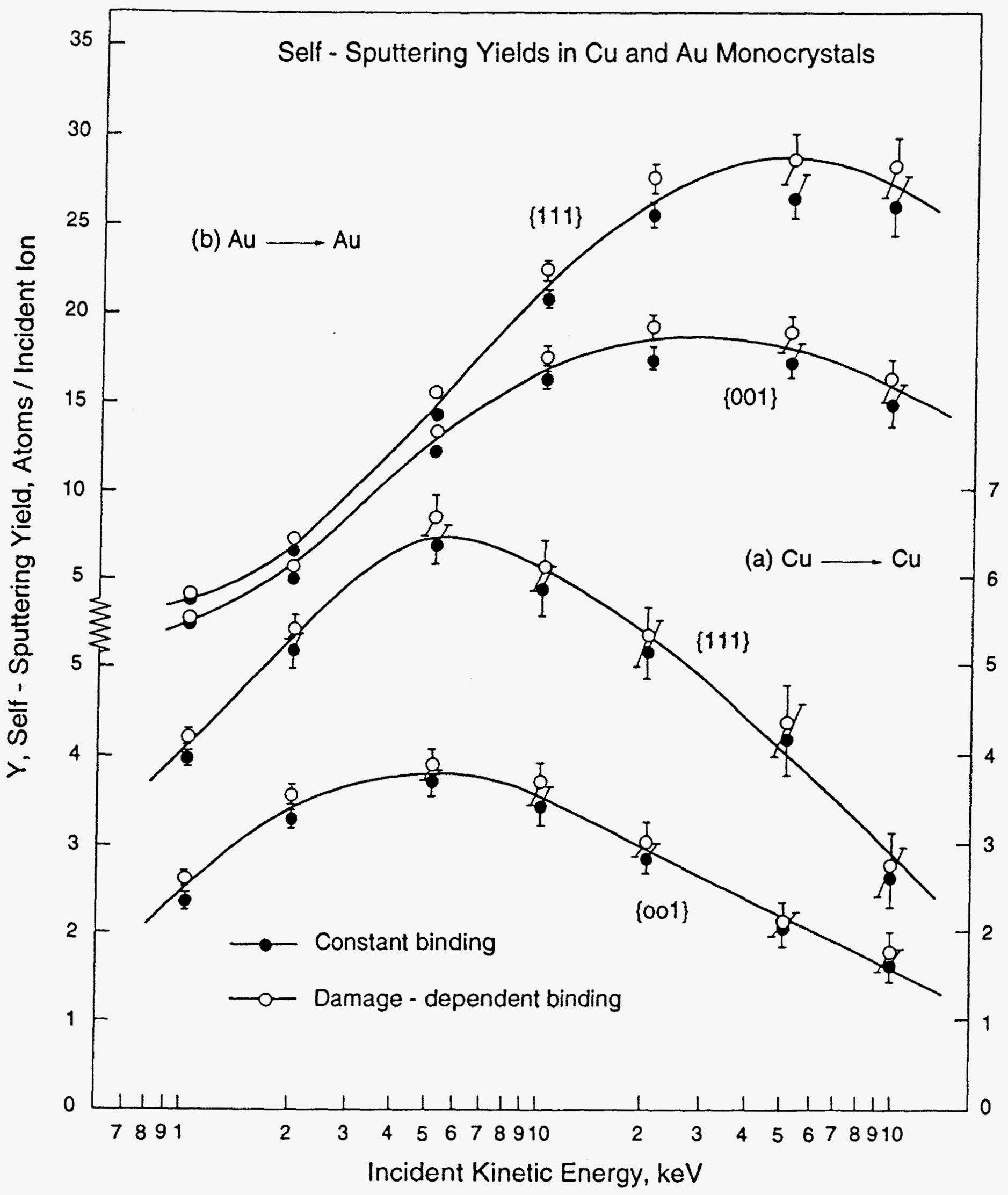




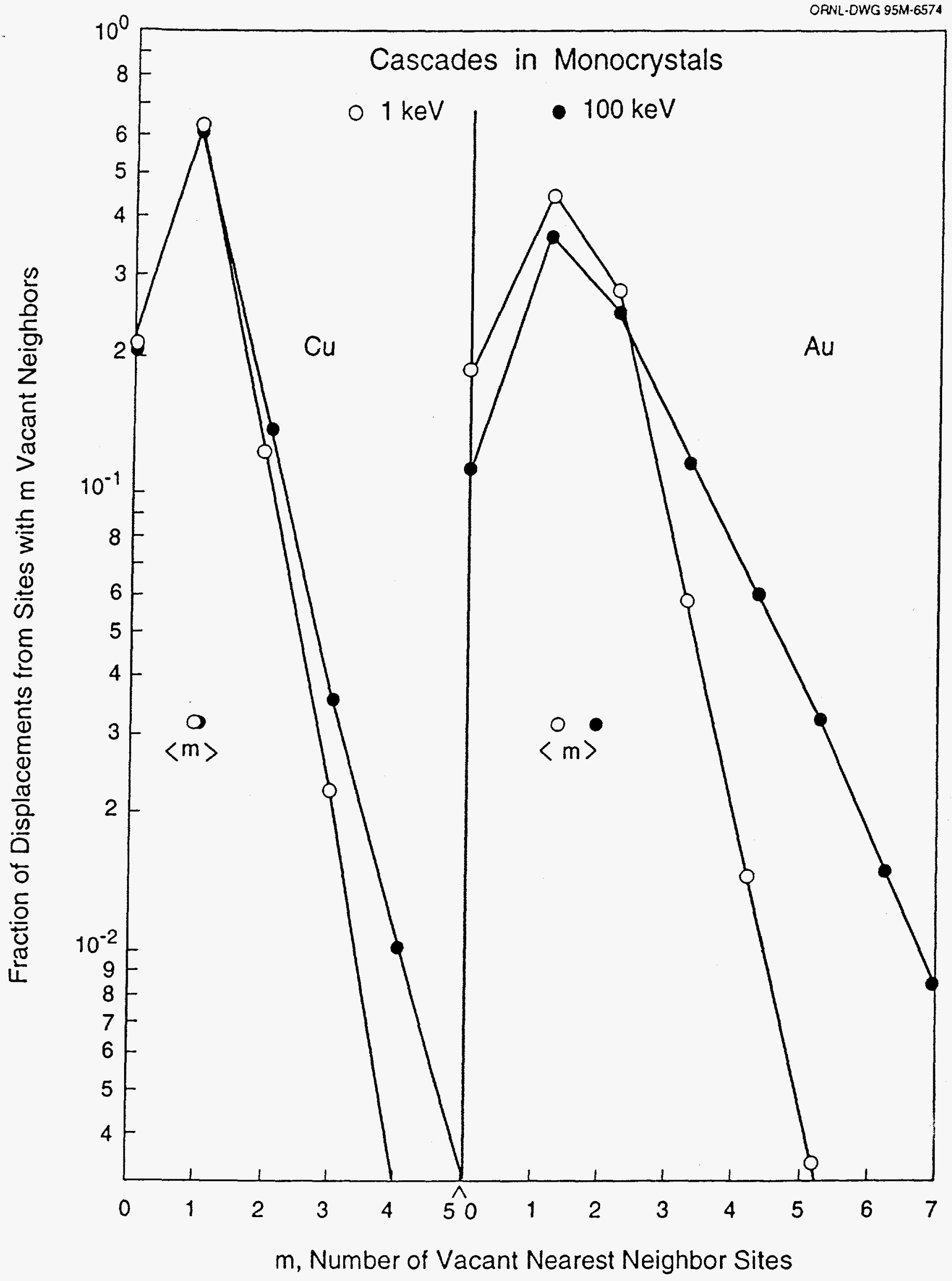




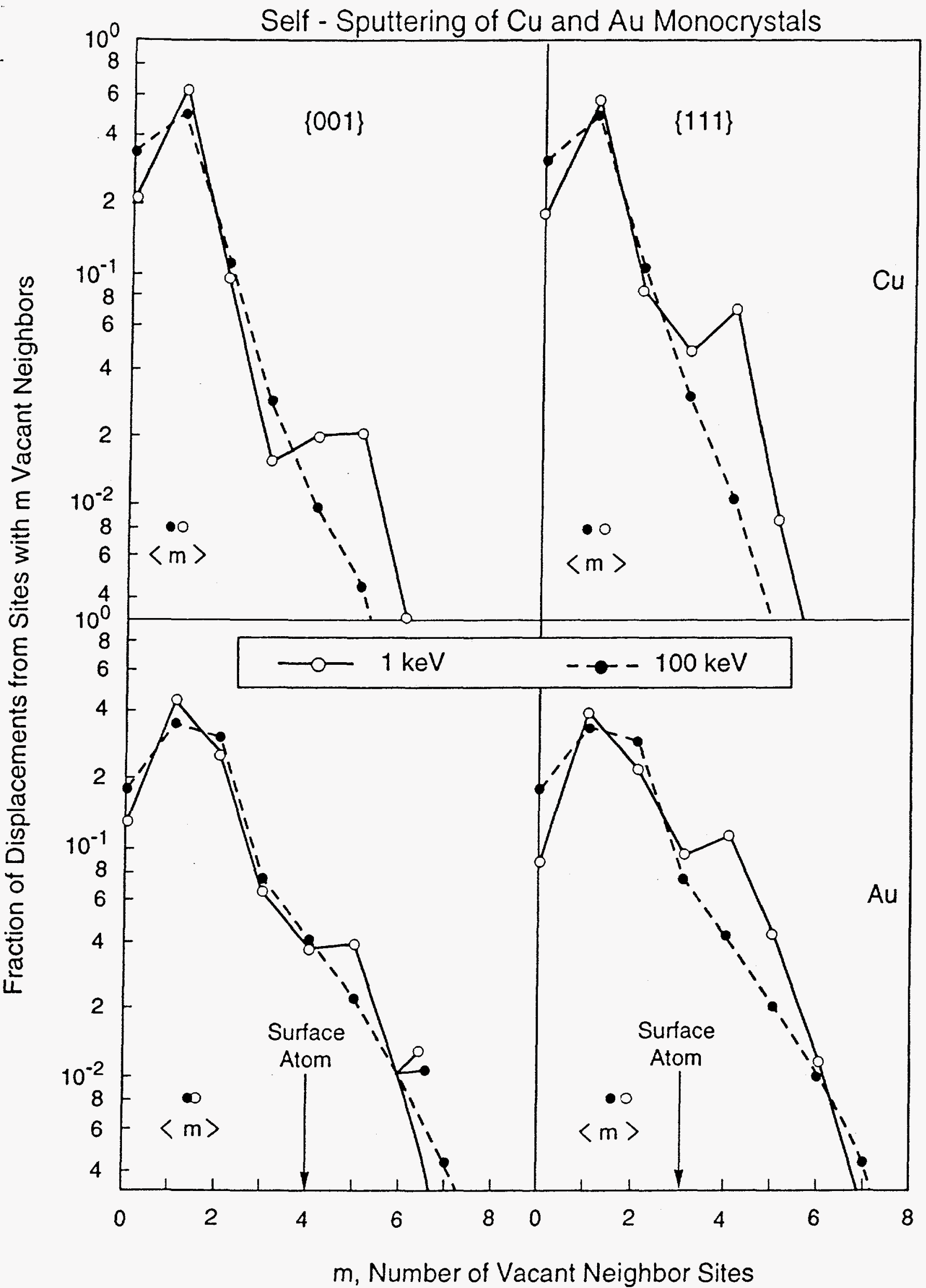




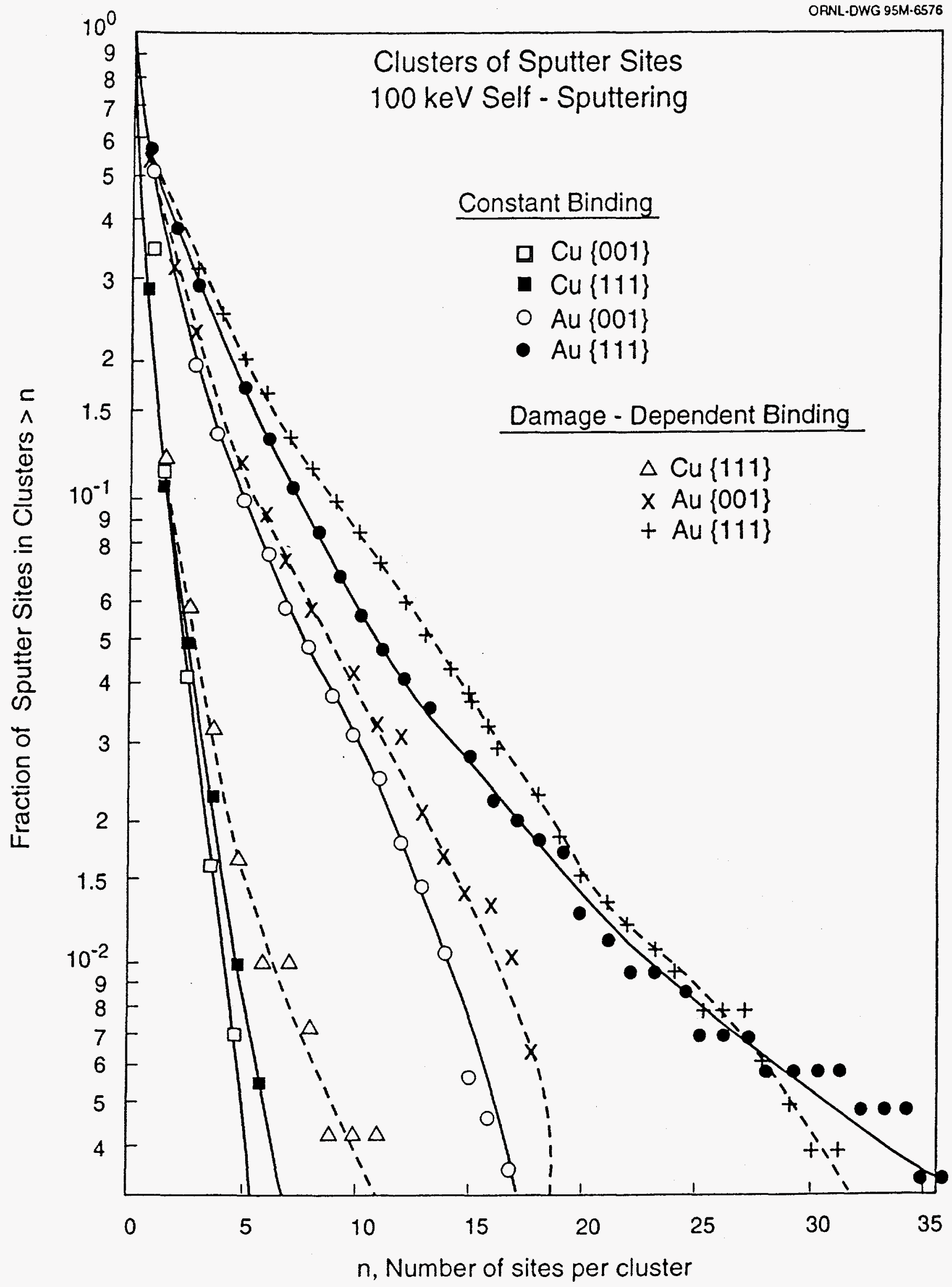




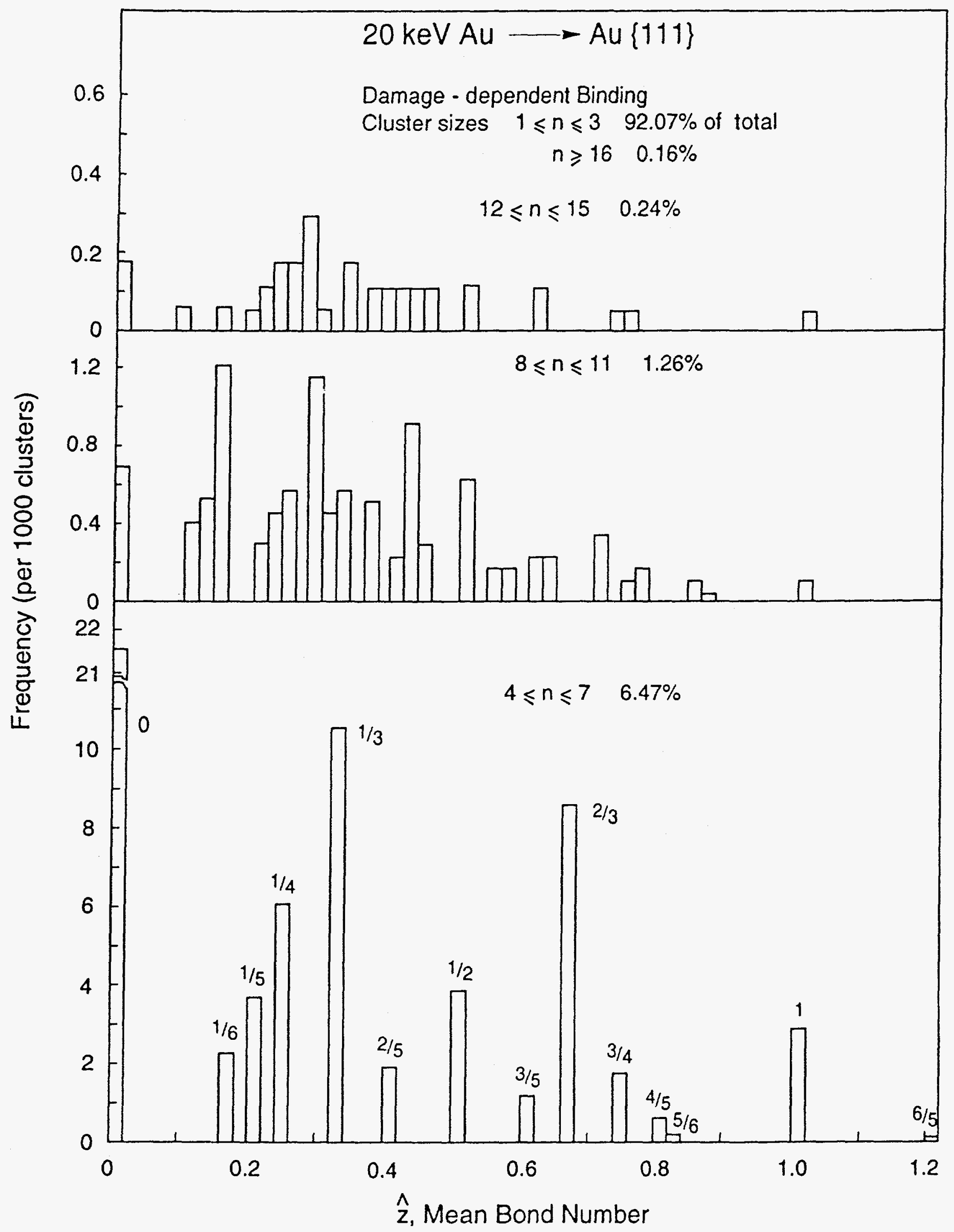

